\section{Sistema de Informações Hospitalares do Sistema Único de Saúde (SIH-SUS): uma avaliação do seu desempenho para a identificação do near miss materno}

\author{
The Hospital Information System of the Brazilian \\ Unified National Health System: a performance \\ evaluation for auditing maternal near miss
}

\author{
Sistema de Información Hospitalaria del Sistema \\ Único de Salud (SIH-SUS): una evaluación de su \\ rendimiento para la identificación de near miss \\ materno
}

Marcos Nakamura-Pereira ${ }^{1}$ Wallace Mendes-Silva 2 Marcos Augusto Bastos Dias 1 Michael E. Reichenheim 3 Gustavo Lobato ${ }^{1}$

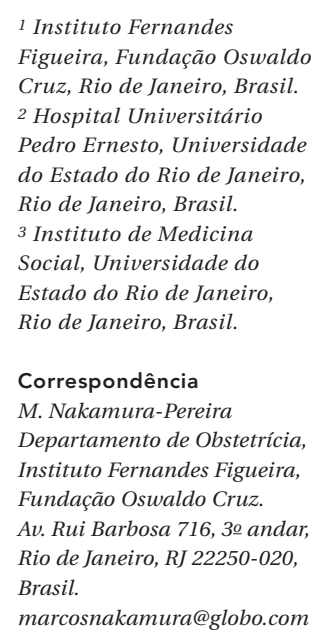

,

\section{Resumo}

Este estudo avaliou o desempenho do Sistema de Informações Hospitalares do Sistema Único de Saúde (SIH-SUS) na identificação de casos de near miss materno ocorridos em hospital do Rio de Janeiro, em 2008. Os casos foram captados pela revisão de todos os prontuários médicos de gestantes ou puérperas internadas na unidade, $e$ a busca por potenciais eventos de near miss na base do SIH-SUS valeu-se de uma lista de procedimentos e códigos da Classificação Internacio-

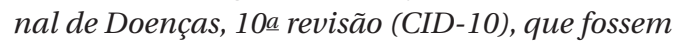
compativeis com esse diagnóstico. A revisão de prontuários identificou 27 casos, enquanto na base do SIH-SUS encontrou-se 70 possíveis ocorrências de near miss. Porém, dessas 70 apenas cinco efetivamente eram casos near miss conforme os prontuários, correspondendo então à sensibilidade de 18,5\% (IC95\%: 6,3-38,1), especificidade de 94,3\% (IC95\%: 92,8-95,6), área sob a curva ROC de 0,56 (IC95\%: 0,48-0,63) e valor preditivo positivo de 10,1\% (IC95\%: 4,7-20,3). Esses achados sugerem que o SIH-SUS não éadequado para o monitoramento dos casos de near miss materno.

Mortalidade Materna; Complicações na Gravidez; Morbidade; Registros Hospitalares; Sistemas de Informação 


\section{Introdução}

A Organização Mundial da Saúde (OMS), em seu último informativo com as estatísticas mundiais de saúde 1 , aponta uma queda de $34 \%$ no número de mulheres que morreram de complicações relacionadas à gestação e ao parto - de 546.000 em 1990 para 358.000 em 2008. Entretanto, esse significativo decréscimo corresponde a menos da metade daquele necessário para o cumprimento de um dos Objetivos do Milênio, especificamente a meta de se reduzir a mortalidade materna em 3/4 entre 1990 e 2015 1. Assim, a mortalidade materna persiste como problema de saúde pública, em especial nos países não desenvolvidos 1,2 .

Contudo, a morte materna representa apenas uma pequena parcela do total de desfechos maternos adversos. As complicações da gravidez podem ser analisadas dentro de um espectro que varia entre a saúde e a morte, em que a gestação pode transcorrer sem quaisquer complicações; apresentar intercorrências clínicas ou cirúrgicas; cursar com morbidade grave; evoluir com risco de vida ou mesmo culminar no óbito materno 3 .

Nesse contexto, surgiu na década de 1990 o conceito de near miss materno para caracterizar os casos em que mulheres apresentaram complicações potencialmente fatais durante gravidez, parto e puerpério, porém escaparam da morte por sorte ou por terem recebido cuidado apropriado e em tempo hábil 4,5 . O progressivo declínio das taxas de mortalidade materna, especialmente nos países desenvolvidos, tornou relevante o estudo do near miss materno. Tendo em vista que as condições que cercam o near miss são bastante semelhantes àquelas que se associam ao óbito materno ${ }^{6}$, e que o near miss apresenta incidência consideravelmente maior, seu estudo pode oferecer conclusões mais robustas sobre a assistência obstétrica prestada em diferentes contextos e, por conseguinte, nortear ações que visem a minimizar a morbimortalidade materna 7,8,9,10.

Ao longo dessas três décadas, o maior entrave para a efetiva consolidação do conceito de near miss materno tem sido a falta de uma demarcação teórico-operacional clara e consensual para o evento 5,6,11,12. Frente a essa dificuldade, em 2009, um grupo de especialistas em saúde materna e perinatal propuseram, sob a chancela da OMS, que o near miss materno seja definido como "uma mulher que quase morre, porém sobrevive à complicação ocorrida na gravidez, parto ou até 42 dias após o fim da gestação" 6 (р. 289), alinhando temporalmente esta definição com a de morte materna 3 . Ainda, esses pesquisadores recomendaram que a definição operacional dos eventos de near miss materno fosse baseada numa lista de 25 critérios clínicos, laboratoriais e de manejo. Dentre os princípios que nortearam a concepção do instrumento, ressalta-se que os itens selecionados visam a captar situações de grave disfunção orgânica e iminente morte materna, evitando assim superestimar a incidência desta condição, e também que seriam critérios passíveis de utilização independentemente das características estruturais de cada unidade de saúde 6 .

Com base nesses conceitos, tem sido sugerido que o monitoramento dos eventos de near miss materno seja realizado rotineiramente por meio de comitês institucionais, ou mesmo por auditorias periódicas ${ }^{6}$. No entanto, ambas as estratégias exigem um sistema de vigilância complexo e dispendioso, e poucos países, ou mesmo instituições, possuem recursos materiais e humanos disponíveis para esse fim. No Reino Unido, por exemplo, a despeito da ênfase dada à necessidade de revisão dos casos de morbidade materna grave por meio de dispositivos como o Sistema deVigilância Obstétrica do Reino Unido (UKOSS) e do Sistema de Vigilância da Morbidade Materna Grave da Escócia 13, a falta de recursos ainda é um entrave importante para a consolidação de uma rotina abrangente de auditoria no âmbito da saúde materna e perinatal 14 .

Frente ao elevado custo operacional para a identificação dos casos de near miss a partir do seguimento prospectivo ou revisão dos prontuários médicos, diversos autores têm proposto a utilização das informações obtidas nas bases de dados administrativas como alternativa para a vigilância dos casos de morbidade materna e near miss 15,16,17,18. Entretanto, a maior parte dessas publicações têm se valido de reflexões teóricas sobre essa estratégia 15,18 , ou projetado incidências de morbidade materna e near miss sem uma avaliação preliminar de sua validade 17 .

No Brasil, o Sistema de Informações Hospitalares do Sistema Único de Saúde (SIH-SUS) é a única fonte de informações sobre a morbidade hospitalar no país 19 . Trata-se de uma base de dados administrativa que armazena as informações das internações hospitalares ocorridas no sistema público de saúde, o qual responde por aproximadamente $70-80 \%$ das internações, e tem seu funcionamento baseado na Autorização de Internação Hospitalar (AIH). Essa deve ser preenchida em todos os casos de internação, mesmo em casos de transferências, e tem como principal finalidade o reembolso financeiro às unidades de saúde pelos serviços prestados 19. Quanto à assistência obstétrica, uma das potenciais vantagens da utilização do SIH-SUS é sua cobertura, tendo em vista que no Brasil os partos ocorrem quase 
que exclusivamente em hospitais, e cerca de $70 \%$ destes são realizados em unidades de saúde públicas ou conveniadas ao SUS 20 .

Em um esforço para estimar a frequência do near miss materno no Brasil, Sousa et al. 15 utilizaram os dados do SIH-SUS para a identificação destes eventos nas capitais brasileiras. Em linhas gerais, embora ressaltando algumas limitações do sistema quando de sua aplicação na pesquisa científica, esses autores reconheceram como promissoras as iniciativas referentes ao monitoramento do near miss materno no Brasil baseando-se nos dados secundários hoje disponíveis. Porém, estudos voltados para a avaliação da adequação do SIH-SUS como ferramenta para a captação da magnitude e da distribuição das doenças na população têm mostrado resultados heterogêneos e limitados por uma série de fatores, tais como a variação na qualidade dos dados; reduzida disponibilidade de informação clínica na AIH; falta de universalidade da cobertura e discrepância na facilidade de acesso entre regiões; utilização regida por regras de financiamento; e demanda influenciada pela oferta 19,21. Nessa linha de investigação crítica, por exemplo, Lobato et al. 22 mostraram recentemente a inadequação do SIH-SUS no monitoramento da doença hemolítica perinatal em nível populacional. Sugerem os autores que a utilização de bases de dados secundários na vigilância epidemiológica seja precedida por um estudo de avaliação da adequação da iniciativa, considerando não somente os agravos a serem rastreados como também o período de tempo e o perfil das unidades de saúde a serem estudadas.

Assim sendo, o presente trabalho propõe uma investigação preliminar do desempenho do SIH-SUS para a captação dos casos de near miss materno ocorridos em uma unidade de saúde pública da cidade do Rio de Janeiro, Brasil. Avaliaremos se essa base de dados, abrangente e de grande porte, pode de fato ser utilizada na vigilância epidemiológica desses eventos.

\section{Métodos}

Este trabalho se baseia na comparação das informações obtidas a partir da revisão das internações ocorridas no Núcleo Perinatal do Hospital Universitário Pedro Ernesto, Universidade do Estado do Rio de Janeiro (HUPE/UERJ), e daquelas disponibilizadas na base de dados do SIH-SUS. Inicialmente, procedeu-se à revisão de todos os sumários de alta referentes às internações ocorridas no Núcleo Perinatal do HUPE/UERJ durante o ano de 2008. Esses sumários estão disponíveis apenas em meio físico e armazenados na própria maternidade do hospital. São voltados para atividades de auditoria e pesquisa e agregam informações acerca da internação, incluindo os diagnósticos obstétricos e os procedimentos médicos realizados. Outros formulários, visando ao faturamento hospitalar, são também confeccionados quando da alta, porém oferecem informação clínica substancialmente reduzida se comparados aos sumários de alta.

Nessa primeira etapa foram selecionados os casos cujos diagnósticos clínicos, exames laboratoriais ou procedimentos realizados sugerissem que a mulher evoluíra apresentando uma ou mais condições potencialmente ameaçadoras à vida, conforme definição da OMS 6 (Tabela 1). Nos casos positivos para ao menos uma dessas condições, o prontuário médico foi subsequentemente revisado. Esses prontuários, também disponíveis apenas em meio físico, foram avaliados após solicitação formal à Seção de Arquivo e Protocolo da instituição. Todas essas atividades foram realizadas por dois dos autores (M.N.P. e W.M.S.), obstetras especialistas em saúde perinatal e treinados para atividades de auditoria relacionada à morbimortalidade materna.

Com base nas informações obtidas nos prontuários médicos selecionados e revisados, os casos foram classificados como near miss segundo os critérios estabelecidos pela OMS 6 (Tabela 2), sendo este considerado o procedimento de referência para a definição diagnóstica de near miss materno ${ }^{6}$. Casos de morbidade materna "não near miss" e óbitos maternos também foram identificados 3,6. Características sociodemográficas, reprodutivas e outras informações acerca da internação e do parto foram também obtidas com base nesses prontuários. Os dados foram compilados em um questionário desenvolvido especificamente para a pesquisa.

A segunda etapa referente à obtenção de dados para a execução do estudo baseou-se na solicitação à Secretaria Municipal de Saúde do Rio de Janeiro das informações completas de todas as AIHs consolidadas na base de dados do SIH-SUS, referentes aos anos de 2008 e 2009. Com base nesse banco de dados, e considerando os objetivos do estudo, algumas restrições foram empregadas: (1) manutenção na base de dados apenas das internações cujas datas de admissão hospitalar tenham ocorrido entre $01 /$ janeiro/2008 e 31/dezembro/2008; (2) restrição às internações ocorridas no HUPE/UERJ por meio do número do hospital no Cadastro Nacional de Estabelecimentos de Saúde (CNES no 2269783); (3) exclusão das solicitações de cobrança (AIHs) rejeitadas; (4) restrição às internações cuja especialidade era Obstetrícia ou cujo diagnóstico principal e/ou secundário fosse do capítulo "O" da Classificação 
Tabela 1

Condições potencialmente ameaçadoras à vida da mulher no ciclo grávido-puerperal *

\begin{tabular}{ll}
\hline Distúrbios hemorrágicos & \multicolumn{1}{c}{ Distúrbios hipertensivos } \\
\hline $\begin{array}{l}\text { Descolamento prematuro de placenta } \\
\text { Placenta acreta, increta ou percreta }\end{array}$ & Pré-eclâmpsia grave \\
Prenhez ectópica & Hipertensão grave \\
Hemorragia pós-parto & Encefalopatia hipertensiva \\
Rotura uterina & Síndrome HELLP ** \\
Outros distúrbios sistêmicos & Indicadores de gravidade de manejo \\
Endometrite & Transfusão sanguínea \\
Edema pulmonar & Acesso venoso central \\
Insuficiência respiratória & Histerectomia \\
Convulsões & Admissão à unidade de tratamento intensivo \\
Sepse & Internação hospitalar prolongada (> 7 dias pós-parto) \\
Choque & Intubação não anestésica \\
Trombocitopenia < 100.000 & Retorno à sala operatória (centro cirúrgico) \\
Crise tireotóxica & Intervenção cirúrgica \\
\hline
\end{tabular}

* Conforme proposição da Organização Mundial da Saúde 5;

** Síndrome relacionada a um quadro de pré-eclâmpsia grave que tenha evoluído com Hemolysis, Elevated Liver Enzymes and Low Platelets.

Tabela 2

Critérios diagnósticos do near miss materno *.

\begin{tabular}{|c|c|c|}
\hline Critérios clínicos & Critérios laboratoriais & Critérios de manejo \\
\hline Cianose aguda & Saturação de $\mathrm{O}_{2}<90 \%$ por $>60 \mathrm{~min}$ & Uso de droga vasoativa contínua \\
\hline Gasping & Lactato $>5$ & Diálise para insuficiência renal aguda \\
\hline Frequência respiratória $>40$ ou $<6 / \mathrm{min}$ & Creatinina $\geq 300 \mu \mathrm{mol} / \mathrm{L}$ ou $3,5 \mathrm{mg} / \mathrm{dL}$ & Reanimação cardiopulmonar \\
\hline $\begin{array}{l}\text { Ausência de consciência e de pulso ou } \\
\text { batimento cardíaco }\end{array}$ & $\begin{array}{l}\text { Ausência de consciência e presença de glicose } \\
\text { e cetoacidose na urina }\end{array}$ & $\begin{array}{l}\text { Transfusão } 5 \text { unidades de concentrados de } \\
\text { hemácias }\end{array}$ \\
\hline Oligúria não responsiva a fluidos ou diuréticos & Trombocitopenia aguda ( $<50.000$ plaquetas) & $\begin{array}{l}\text { Intubação e ventilação } 60 \text { min, não relacionada } \\
\text { à anestesia }\end{array}$ \\
\hline Acidente vascular cerebral & $\mathrm{pH}<7,1$ & Histerectomia puerperal \\
\hline Choque & Bilirrubina $>100 \mu \mathrm{mol} / \mathrm{L}$ ou $>6,0 \mathrm{mg} / \mathrm{dL}$ & \\
\hline Perda de consciência $\geq 12 \mathrm{~h}$ & $\mathrm{PaO}_{2} / \mathrm{FiO}_{2}<200 \mathrm{mmHg}$ & \\
\hline \multicolumn{3}{|l|}{ Distúrbios da coagulação } \\
\hline \multicolumn{3}{|l|}{ Icterícia na presença de pré-eclâmpsia } \\
\hline Convulsão não controlada/Paralisia total & & \\
\hline
\end{tabular}

* Conforme a proposição da Organização Mundial da Saúde 5, uma mulher apresentando qualquer das condições acima e que sobreviva a complicações que tenham ocorrido durante a gestação, parto ou em até 42 dias depois de terminada a gestação deve ser considerada um caso de near miss materno.

Internacional de Doenças - 10ạ revisão (CID-10); (5) exclusão dos casos de morte materna, visto que o objetivo precípuo da pesquisa concerne apenas às mulheres que, a despeito de graves complicações, não evoluíram com óbito durante a internação obstétrica no HUPE/UERJ. Assim, o banco de dados processado baseando-se no SIHSUS teve o mesmo universo amostral empregado para a revisão dos sumários de alta hospitalar.

A avaliação do desempenho do SIH-SUS para a identificação dos casos de near miss materno ocorridos no ano de 2008 no HUPE/UERJ com- 
preendeu estratégias complementares e independentes. Inicialmente, os nomes e a data de internação dos casos de near miss identificados pela revisão de prontuários foram utilizados para uma busca individual na base do SIH-SUS. Além de uma estimativa da sensibilidade do SIH-SUS para a identificação dos casos de near miss, essa etapa teve como propósito uma análise predominantemente qualitativa das razões que justificassem eventuais ausências na base de dados de algum evento de near miss.

Concomitantemente, os dados da base do SIH-SUS foram escrutinados para a seleção das AIH cujos campos "Diagnóstico Principal", "Diagnóstico Secundário" ou "Procedimento Realizado" apresentassem codificações compatíveis com um evento de near miss materno. Similarmente à estratégia já adotada por outros autores ${ }^{17}$, para esse propósito, foi elaborada uma lista de procedimentos e de códigos de doenças, conforme o CID-10, que se aproximasse do conjunto de 25 itens do instrumento diagnóstico de near miss materno proposto pela OMS (Tabela 3) 23. Esse procedimento teve o intuito de identificar eventuais situações em que casos de near miss seriam identificados apenas pelo SIH-SUS, e não pela revisão de prontuários, e as razões que contribuíram para isto. Por fim, a última etapa da análise compreendeu a avaliação das propriedades diagnósticas do SIH-SUS para identificação dos eventos de near miss materno.

Os formulários referentes aos casos de near miss identificados na revisão dos prontuários do HUPE/UERJ foram codificados e digitados empregando-se o programa Epi Info (Centers Disease Control and Prevention, Atlanta, Estados Unidos). O processamento dos dados e as análises estatísticas foram conduzidos no programa Stata 12 (Stata Corp., College Station, Estados Unidos), e todas as estimativas, incluindo a sensibilidade, especificidade, valor preditivo positivo (VPP), valor preditivo negativo (VPN) e acurácia foram obtidas assumindo um intervalo de $95 \%$ de confiança (IC95\%). No que tange ao VPP e VPN, também serão projetadas suas estimativas conforme diferentes magnitudes do near miss materno ${ }^{11}$.

O projeto foi aprovado pelo Comitê de Ética em Pesquisa do HUPE/UERJ (registro no 2751/2010) e do Instituto Fernandes Figueira (registro no 0001/11).

\section{Resultados}

Foram revisados 1.170 sumários de internação referentes às admissões hospitalares ocorridas no Núcleo Perinatal do HUPE/UERJ no ano de 2008, sendo selecionados para a revisão com- pleta dos prontuários médicos 228 casos que apresentavam condições potencialmente ameaçadoras à vida da mulher. Desses, 221 tiveram os prontuários efetivamente escrutinados e sete prontuários não foram encontrados. Após revisão das informações contidas nos registros médicos, foram confirmados oito óbitos maternos, 130 mulheres cujos quadros clínicos eram compatíveis com morbidade materna não near miss e 27 casos em que efetivamente o diagnóstico de near miss materno pôde ser confirmado (Figura 1). Tendo em vista que as informações disponibilizadas pelo Sistema de Informações sobre Nascidos Vivos (SINASC. http:tabnet.datasus.gov.br/ CGI/sinasc/dados/indice.htm, acessado em 25/ Mar/2011) apontam que houve 812 nascidos vivos no HUPE/UERJ no ano de 2008, a razão de near miss materno foi de 33,2 por mil nascidos vivos ${ }^{6}$.

Em relação às características sociodemográficas das 27 mulheres diagnosticadas com near miss materno, a idade média foi de 26 anos (IC95\%: 16-37); 14 delas (51,8\%) não tinham o ensino médio completo; e apenas $7(25,9 \%)$ eram casadas ou viviam em união estável. Ainda, 10 (37\%) eram nulíparas, 11 (40,7\%) primíparas e $6(22,3 \%)$ secundíparas ou multíparas por ocasião da admissão. Sete $(25,9 \%)$ apresentavam história de aborto, 10 (37\%) tinham parto cesáreo prévio e $2(7,4 \%)$ cursavam com gestação gemelar. Apenas um caso de near miss $(3,7 \%)$ foi admitido após o parto em outra unidade hospitalar e, dentre aquelas internadas ainda na gestação ( $n=26 ; 96,3 \%)$, a idade gestacional média na internação foi de 30 semanas (IC95\%: 7-40). Sete mulheres $(25,9 \%)$ receberam alta hospitalar ainda como gestantes e, dentre as $19(70,4 \%)$ cujos partos ocorreram no HUPE/UERJ, a idade gestacional média ao nascimento foi de 33,6 semanas (IC95\%: 22-40), com 11 (57,8\%) partos cesáreos e $8(42,1 \%)$ vaginais. O tempo de internação médio foi de 15,2 dias (IC95\%: 3-50).

Houve predominância dos quadros de hemorragia ( $\mathrm{n}=11 ; 40,7 \%)$ e das síndromes hipertensivas ( $n=8 ; 29,6 \%$ ) como os principais determinantes da ocorrência do near miss materno nessa casuística. Em relação aos itens diagnósticos empregados, plaquetopenia aguda $(40,7 \%)$, choque $(37 \%)$ e creatinina $\geq 3,5 \mathrm{mg} / \mathrm{mL}(18,5 \%)$ foram aqueles mais comumente encontrados, enquanto cianose aguda, gasping, perda da consciência e ausência de pulso, lactato $>5$, perda da consciência com presença de glicose e cetoácidos na urina e ressuscitação cardiopulmonar foram itens não encontrados em nenhum dos 27 casos de near miss.

Na avaliação inicial do desempenho do SIHSUS, a busca nominal das mulheres identificadas 
Tabela 3

Caracterização dos diagnósticos e procedimentos, correlacionados aos critérios de near miss materno da Organização Mundial da Saúde (OMS) 5, utilizados na identificação dos casos de near miss no Sistema de Informação Hospitalar do SUS (SIH-SUS).

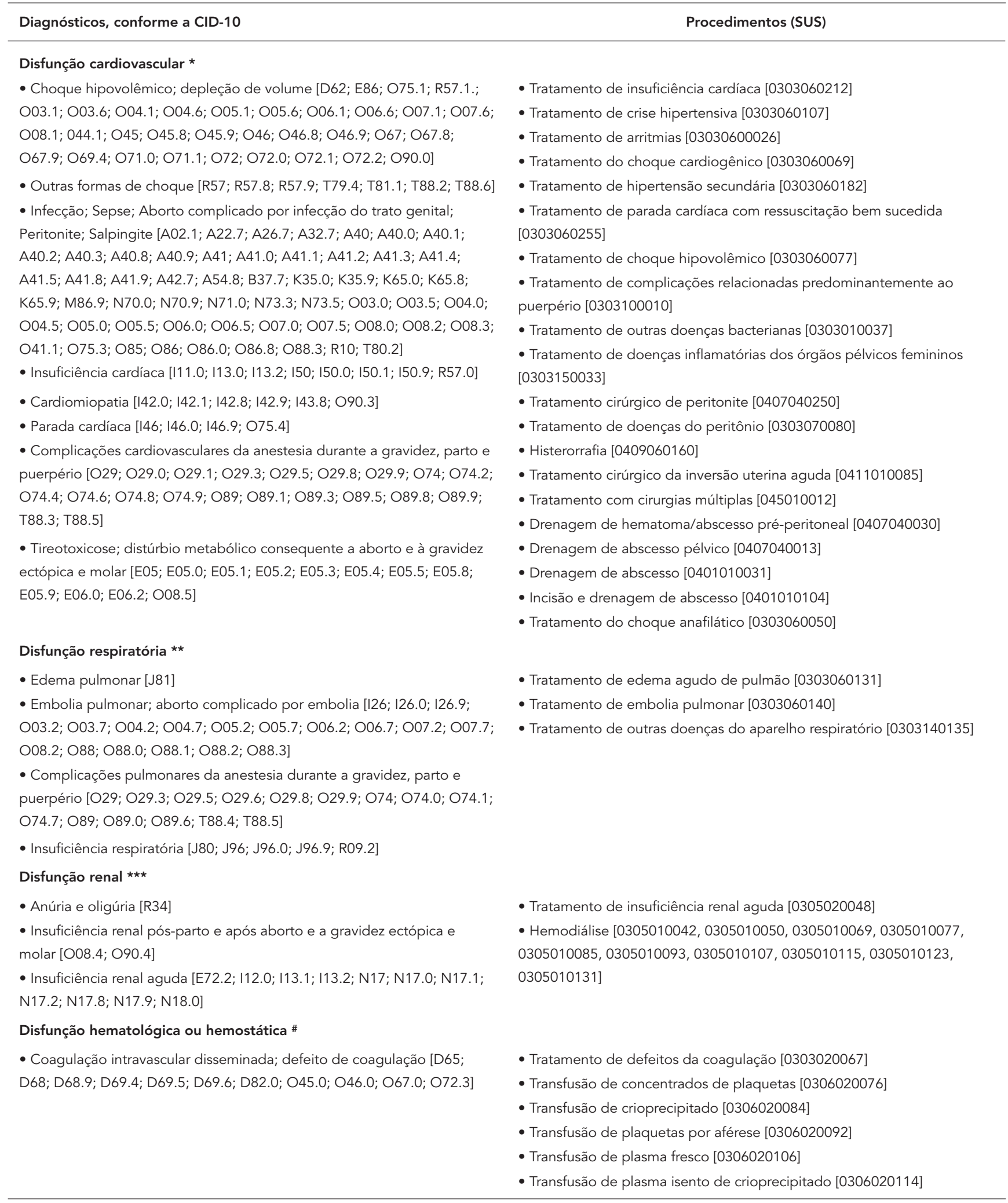

(continua) 


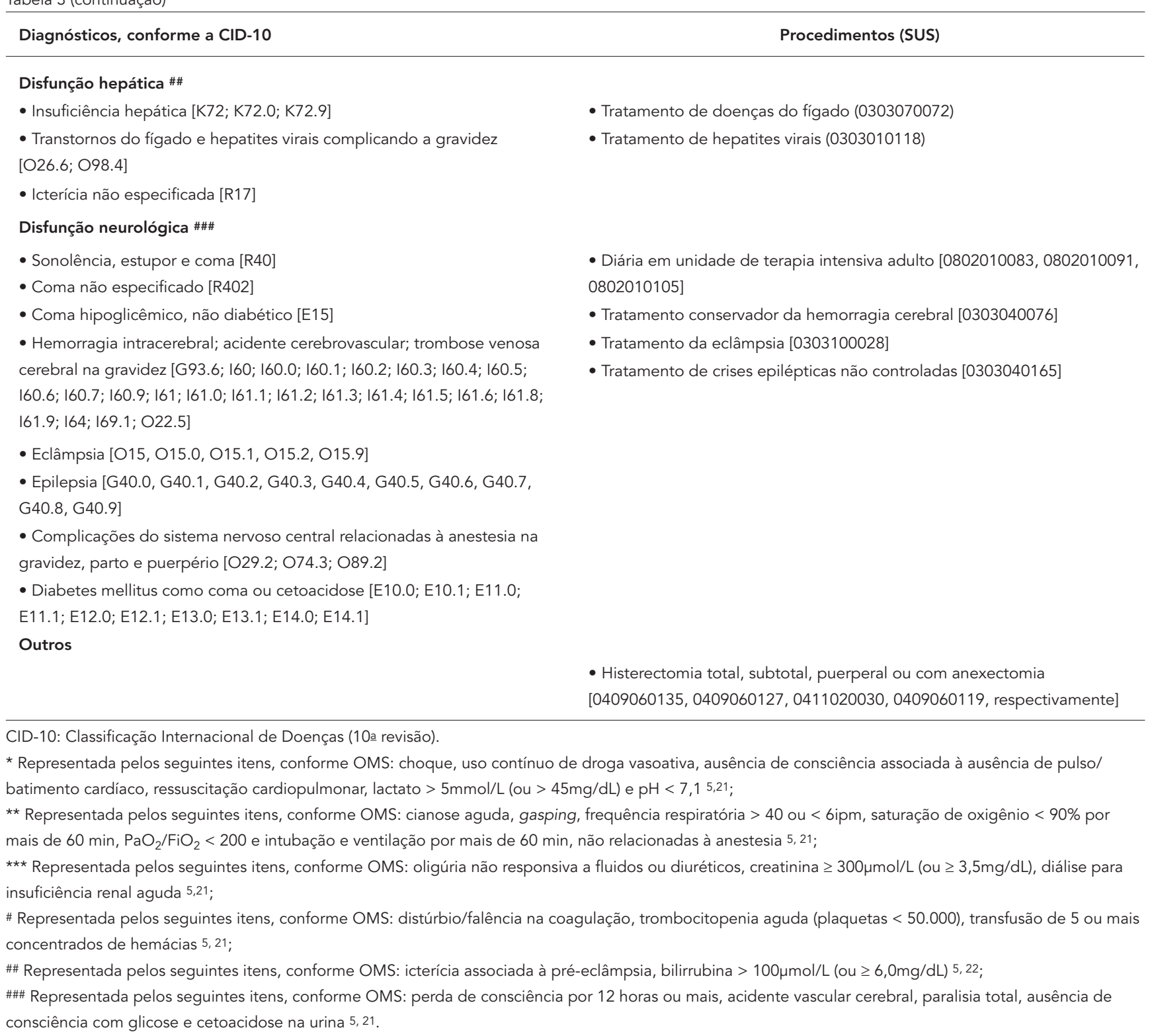

como near miss na revisão dos prontuários no sistema revelou que apenas 16 (59,3\%) dos casos estavam consolidados no banco de dados, independentemente dos campos diagnósticos e de procedimento serem compatíveis ou não com o diagnóstico de near miss. Acerca da ausência no sistema dos outros 11 casos (40,7\%), foi possível confirmar que ao menos quatro $(36,3 \%)$ destas ausências decorreram do não faturamento da AIH em razão do hospital ter excedido o limite percentual de cesarianas para o período em questão e, dentre as sete restantes, cinco também tiveram partos cesáreos, totalizando $81,8 \%$ de cesarianas entre os casos de near miss cujas AIHs não estavam consolidadas no SIH-SUS.
Os critérios empregados para selecionar as internações presentes na base do SIH-SUS, resultaram em apenas 666 internações obstétricas sem óbito ocorridas no HUPE/UERJ no ano de 2008, o que difere do número encontrado na revisão dos sumários de internação hospitalar. Dessas 666 internações, a lista de códigos da CID-10 e procedimentos listados como proxies para o diagnóstico de near miss (Tabela 3) identificou 70 casos, o que resultaria em uma razão de near miss materno de 86,2 por mil nascidos vivos, aproximadamente 2,5 vezes maior do que aquela encontrada na revisão dos prontuários. Entretanto, desses 70 casos selecionados no SIH-SUS, apenas cinco efetivamente corres- 
Fluxograma das etapas para identificação dos casos de near miss materno ocorridos no Hospital Universitário Pedro Ernesto, Universidade do Estado do Rio de Janeiro, em 2008, com base na revisão dos prontuários médicos.

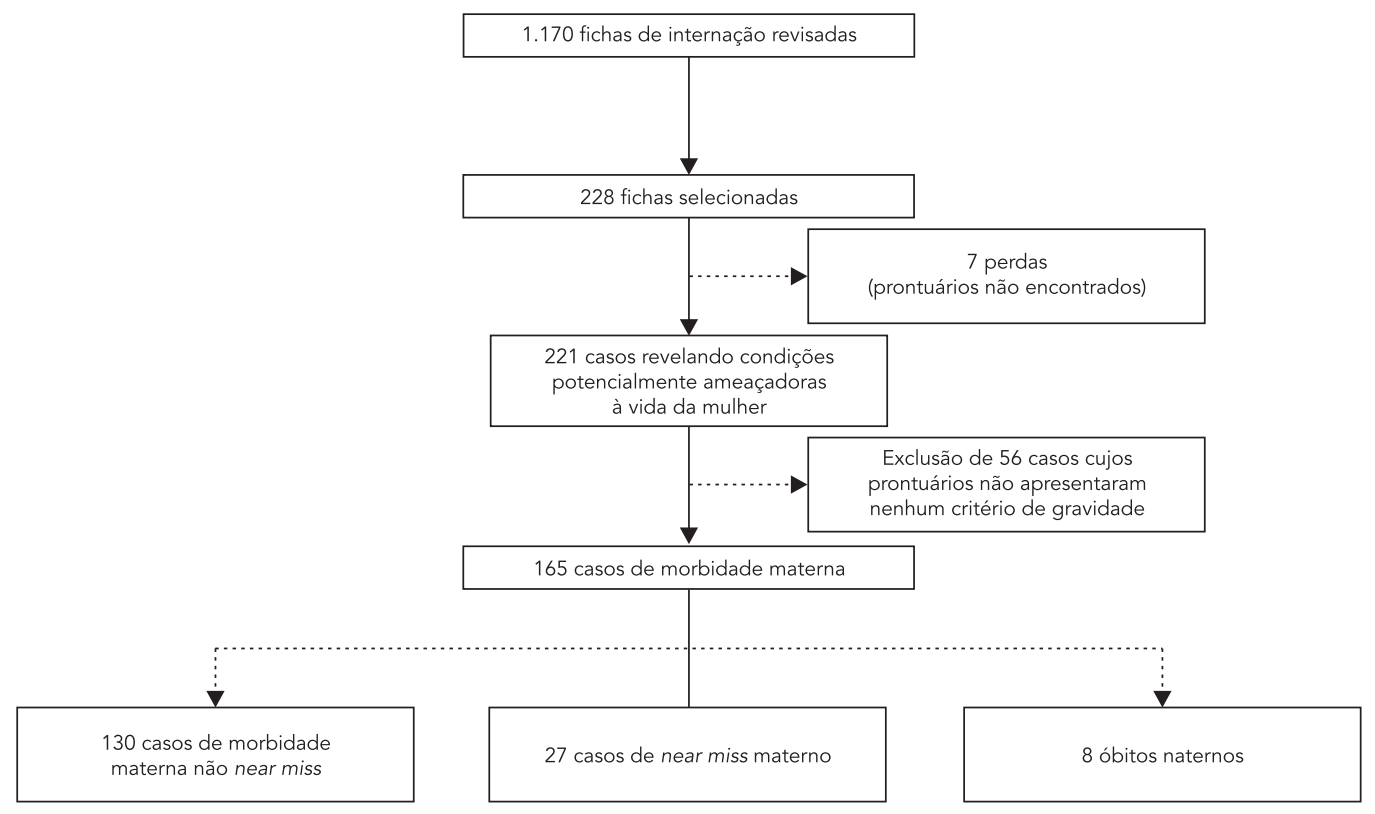

pondem a eventos de near miss materno conforme a revisão dos prontuários. Assim, a sensibilidade do SIH-SUS para captar os casos de near miss ocorridos na unidade de saúde foi de $18,5 \%$ (IC95\%: 6,3-38,1), com especificidade de 94,5\% (IC95\%: 92,8-95,6) e acurácia de 56,4\% (IC95\%: $48,9-63,9)$. No que tange às estimativas referentes aos valores preditivos positivos, no hospital em questão, onde a razão de near miss materno foi de 33,2 por mil nascidos vivos, o VPP de 10,1\% (IC95\%: 4,7-20,3) e o VPN de 97,1\% (IC95\%: 96,697,6).

A Tabela 4 traz as estimativas projetadas para os VPP e VPN do SIH-SUS na identificação do near miss materno conforme diferentes magnitudes do agravo 11. Observa-se que, para incidências variando entre 1 e 110 casos por mil nascidos vivos, o VPP variou de $0,3 \%$ a $28,7 \%$ e o VPN, de $90,4 \%$ a $99,9 \%$

\section{Discussão}

Diante do exposto, o SIH-SUS não é uma alternativa válida para a captação dos casos de near miss materno ocorridos no HUPE/UERJ. Em linhas gerais, sua baixa sensibilidade comprome- te a acurácia da estratégia, a qual se apresentou pouco acima de $50 \%$. No que tange aos valores preditivos, altos valores de VPN poderiam ser antecipados, visto tratar-se de evento relativamente raro. Contudo, há de ressaltar que o VPP do SIH-SUS para a identificação do near miss materno é muito baixo, mesmo em cenários onde a magnitude do evento alcança seus maiores patamares ${ }^{11}$. Assim, sua utilização nesse contexto não parece adequada, independentemente do perfil assistencial e de morbimortalidade da(s) unidade(s) de saúde em questão.

Uma das razões para a precária sensibilidade do SIH-SUS como estratégia alternativa para o monitoramento do near miss materno parece ser o limite percentual de cesarianas estipulado para o reembolso pelo SUS 14,15,16,17,18, que no Estado do Rio de Janeiro é de $27 \%$ de partos cesáreos por unidade de saúde 28 . Segundo os achados do presente estudo, a não consolidação no SIH-SUS das AIH referentes a pelo menos quatro $(14,8 \%)$ dos 27 eventos de near miss ocorreu em virtude desse limite. Contudo, esses números podem ser ainda maiores e chegar a nove casos $(33,4 \%)$, visto que, dentre os 11 casos de near miss cujas AIH não foram consolidadas no SIH-SUS, foi predominante o parto cesáreo $(n=9 ; 81,8 \%)$. 
Tabela 4

Valores preditivos positivo (VPP) e negativo (VPN) do Sistema de Informações Hospitalares do SUS (SIH-SUS), na captação dos casos de near miss materno, conforme diferentes incidências do evento.

\begin{tabular}{lcccc}
\hline Incidência * & \multicolumn{2}{c}{ VPP } & VPN & IC95\% \\
\hline 1 & $\%$ & IC95\% & $\%$ & $99,9-99,9$ \\
10 & 0,3 & $0,1-0,7$ & 99,9 & $99,0-99,3$ \\
30 & 3,1 & $1,4-7,0$ & 99,1 & $96,9-97,8$ \\
50 & 9,1 & $4,2-18,7$ & 97,4 & $94,8-96,3$ \\
90 & 14,6 & $7,0-28,1$ & 95,7 & $92,8-94,9$ \\
110 & 19,7 & $9,7-35,9$ & 93,9 & $90,7-93,3$ \\
\hline
\end{tabular}

* Incidência de near miss materno (por 1.000 nascidos vivos).

Avaliando a qualidade da informação sobre o parto no SIH-SUS no Município do Rio de Janeiro entre 1999 e 2001, Bittencourt et al. 20 encontraram sub-registro de 11,9\% de $6.835 \mathrm{mu}$ lheres internadas em maternidades conveniadas ao SUS. Dessas internações não registradas no sistema, observou-se que a principal razão foi a não emissão da AIH e que havia elevado percentual de cesáreas $(77,2 \%)$ entre as AIH não emitidas, o que, segundo a conclusão dos autores, pode estar relacionado ao cumprimento das portarias que limitam o número de cesarianas. Esses dados parecem corroborar a hipótese de que as portarias que estipulam o percentual máximo de cesarianas nas unidades de saúde contribuem para o fraco desempenho do SIH-SUS na identificação dos casos de near miss materno, visto que estas mulheres estão mais propensas ao parto cirúrgico 29 .

Outra questão que parece ter contribuído para a ineficiência do SIH-SUS para a recuperação dos casos de near miss nessa casuística foi a confiabilidade das informações presentes nos campos diagnósticos e de procedimentos. Embora alguns autores já tenham identificado elevada concordância dos dados referentes aos diagnósticos codificados ${ }^{30}$ e o tipo de parto ${ }^{20}$, no presente estudo notou-se a utilização de alguns diagnósticos inapropriados quando do preenchimento das AIH. Por exemplo, uma internação com quadro de hemorragia pós-parto seguida de histerectomia puerperal foi faturada como neoplasia de comportamento incerto ou desconhecido do útero. Em outro caso, uma gestação com mais de vinte semanas, interrompida por fígado gorduroso agudo/síndrome HELLP, teve como diagnóstico principal aborto por razões médicas e legais - incompleto, sem complicações. Pos- sivelmente, nessas situações, houve um esforço para a compatibilização entre o diagnóstico principal e o procedimento realizado ("laparatomia exploradora" e "curetagem pós-abortamento/ puerperal", respectivamente), conforme a Portaria SAS no 579, de 20 de dezembro de 2001 31, que determina a rejeição das AIH quando esta compatibilidade não for verificada. Esses exemplos refletem a lógica inerente do SIH-SUS, voltada, sobretudo, ao reembolso da assistência hospitalar e não à vigilância epidemiológica ${ }^{18,22}$. Ademais, em algumas situações, a falta de concordância entre os diagnósticos clínicos e aqueles consolidados no SIH-SUS, parecem ter decorrido do preenchimento inadequado dos formulários que servem de base para o preenchimento das AIH. Apesar de cinco $(31,2 \%)$ dos 16 casos de near miss materno cujas AIH estavam presentes na base de dados serem relativos a quadros de hemorragia obstétrica, não houve registro de diagnósticos ou procedimentos compatíveis com esta complicação em nenhum deles, tanto no sumário de alta quanto na AIH.

Igualmente relevante foi a pouca utilização do campo de diagnóstico secundário quando do preenchimento das AIH. Dentre os 16 casos de near miss materno que tiveram AIH consolidadas na base do SIH-SUS, apenas sete $(43,7 \%)$ apresentavam codificações referentes ao campo de diagnóstico secundário. A lacuna no preenchimento desse campo diagnóstico não parece restrita a esse contexto, já tendo sido referido por outros autores 22. $\mathrm{O}$ achado também parece ter relação com a função precípua da base do SIHSUS, de cunho administrativo e voltada para o pagamento às unidades de saúde pela assistência médica prestada, visto que o preenchimento desse campo não é obrigatório para a aceitação 
da AIH e pagamento às unidades de saúde pela assistência médica prestada.

Um obstáculo adicional para a utilização do SIH-SUS no monitoramento dos casos de near miss materno em nível populacional concerne à dificuldade de se correlacionar os critérios da OMS com os diagnósticos listados no CID-10 e a lista de procedimentos definidos pelo SUS, o que em grande parte explica o baixo valor preditivo positivo $(7,14 \%)$ da estratégia. Muitos dos procedimentos e códigos da CID-10 selecionados nesta pesquisa parecem ter pouca equivalência em relação aos critérios clínicos, laboratoriais ou de manejo propostos pela OMS, mas a não inclusão de grande parte dos procedimentos e códigos acarretaria em uma sensibilidade ainda menor do SIH-SUS. Da mesma forma, a inclusão de campos referentes a procedimentos especiais que porventura pudessem informar sobre o uso de drogas vasoativas, transfusão de hemoderivados ou ventilação assistida não teriam uma estrita concordância com os critérios da OMS, os quais se baseiam na quantidade utilizada ou no tempo de utilização desses procedimentos. Assim, sua utilização poderia inclusive acarretar uma piora do VPP da estratégia. Cabe reiterar que a metodologia empregada no presente estudo, especialmente no que tange à seleção desses procedimentos e códigos da CID-10, foi similar àquela adotada por outros autores na Austrália, no Brasil e no Canadá 16,17,32.

Os resultados aqui apresentados referem-se a um hospital universitário, com especificidades em relação à sua clientela e aos procedimentos médicos e administrativos adotados. Nesse sentido, a generalização dos achados e conclusões para outros nosocômios, em diferentes contextos, deve ser vista de forma cautelosa, visto que os estabelecimentos de saúde podem diferir substancialmente nas suas práticas administrativas relacionadas ao preenchimento e emissão de AIH. De qualquer forma, esses resultados e sua interpretação estão de acordo com aqueles obtidos em pesquisas anteriores, referentes a outras doenças e outras instituições ${ }^{23}$, e refletem dificuldades inerentes ao emprego do $\mathrm{SIH}$ SUS para estimar e monitorar doenças em nível populacional. Deve-se também ressaltar que as dificuldades de vigilância do near miss materno a partir de bases de dados administrativas não são exclusivas do sistema brasileiro. No Canadá, Allen et al. 18 também apontam vários entraves à detecção adequada dos casos de morbidade materna grave, tais como diferenças locais na coleta de dados, registro parcial das informações nas bases administrativas e inadequação dos critérios de definição do near miss.
Alguns aprimoramentos no registro e fluxo de informações do SIH-SUS poderiam contribuir para melhorar o desempenho dessa base de dados na vigilância epidemiológica. Por exemplo, os entraves relacionados à codificação da AIH por profissionais administrativos não treinados poderiam ser minimizados 21 . A codificação feita por profissionais especializados, no momento da alta e, sempre que possível, fazendo uso do campo do diagnóstico secundário, deve ser encorajada. Ainda, estratégias que visem a evitar a perda de informação acarretada pela não emissão, por parte dos hospitais, das AIH referentes a partos cesáreos que ultrapassem o limite de reembolso do SIH-SUS seriam interessantes no âmbito da vigilância em saúde materno-infantil. Da mesma forma, relatórios que discriminem casos em que a rejeição da AIH correspondente tenha como uma das motivações o alcance do patamar mensal máximo preconizado para cesarianas seriam úteis para a captação de complicações grávidopuerperais. Em seu formato atual, a revisão das AIH glosadas na base do SIH-SUS é um processo excessivamente oneroso e implica opções metodológicas em que a validade da informação pode estar comprometida. Uma mesma $\mathrm{AIH}$, referente a um único caso clínico, pode ser rejeitada diversas vezes até ser enfim aceita, ou mesmo acabar por ser definitivamente rejeitada. Considerando que todas as "entradas" das AIH efetivamente rejeitadas estão presentes no banco, cada uma dessas "rejeições" deveria ser avaliada individualmente e feita a opção pela inclusão de uma destas "entradas". Por exemplo, considerar a primeira apresentação poderia trazer problemas, visto que essa rejeição pode ter acontecido em função da ausência de informações importantes para o estudo. E a inclusão da última apresentação também poderia acarretar vieses, na medida em que a instituição, na tentativa de ter a AIH aceita e receber o reembolso, poderia adaptar ou mesmo mudar as informações da AIH, comprometendo a validade da informação. De qualquer forma, os resultados deste trabalho apontam que, no contexto da pesquisa, mesmo com a inclusão das AIH rejeitadas a sensibilidade do SIH-SUS na vigilância de casos de near miss não alcançaria $50 \%$, sugerindo que a rejeição de $\mathrm{AIH}$ não seria isoladamente a responsável pelo fraco desempenho do SIH-SUS na vigilância do near miss materno.

Em síntese, esses achados não significam que a base de dados do SIH-SUS não possa ser utilizada em ações de vigilância relacionadas à saúde materna. O uso de um grupo de diagnósticos, relacionados a complicações obstétricas e que guardem correspondência com o CID-10, notadamente aquelas situações classificadas co- 
mo potencialmente ameaçadoras à vida (Tabela 1), pode ser uma opção interessante para a vigilância epidemiológica global da ocorrência de algumas intercorrências, inclusive permitindo a implantação de ações de saúde voltadas para sua redução. Ainda, a utilização de técnicas de relacionamento probabilístico de dados, seja com outras bases de dados secundários ou mesmo com bases de dados locais, institucionais, pode vir a colaborar e otimizar a performance do SIHSUS como ferramenta de monitoramento dos agravos relacionados ao ciclo grávido-puerperal em nosso meio $33,34,35,36$. No entanto, ainda que os resultados deste estudo se restrinjam a uma unidade de saúde e a um único ano, especificamente para o monitoramento dos casos de near miss materno o emprego da base do SIH-SUS não parece adequado.

\section{Resumen}

Este estudio evaluó el desempeño del Sistema de Información Hospitalaria del Sistema Único de Salud (SIH-SUS) en la identificación de casos de near miss materno en un hospital de Río de Janeiro en 2008. Los casos se obtuvieron mediante revisión de los historiales clínicos de las embarazadas y puérperas ingresadas en la unidad, y la búsqueda de posibles eventos near miss en la base del SIH-SUS se basó en una lista de procedimientos y códigos de la Clasificación Internacional de Enfermedades (CIE-10) que son compatibles con este diagnóstico. Una revisión retrospectiva de historiales clínicos identificó 27 casos, mientras que la base de la SIH-SUS localizó 70 posibles casos con este diagnóstico. Sin embargo, sólo cinco de los 70 casos fueron en realidad casos de near miss, por ello, la sensibilidad correspondiente fue de un 18,5\% (IC95\%: 6,3-38,1), una especificidad del 94,3\% (IC95\%: 92,8-95,6), el área bajo la curva ROC de un 0,56 (IC95\%: 0,48-0,63) y el valor predictivo positivo de un 10,1\% (IC95\%: 4,7-20,3). Estos hallazgos sugieren que SIH-SUS no es adecuado para el seguimiento de near miss.

Mortalidad Materna; Complicaciones del Embarazo; Morbilidad; Registros de Hospitales; Sistemas de Información

\section{Colaboradores}

M. Nakamura-Pereira participou de todas as etapas da pesquisa e da redação do artigo. W. Mendes-Silva colaborou na coleta de dados e na redação do manuscrito. M. A. B. Dias participou da concepção da pesquisa e da redação do artigo. M. E. Reichenheim participou da concepção do estudo e da redação do manuscrito. G. Lobato participou da concepção do estudo, da análise dos dados e da redação do artigo.

\section{Agradecimentos}

M. E. Reichenheim foi parcialmente apoiado pelo CNPq, processo no 301221/2009-0. Os autores agradecem aos funcionários da Secretaria $\mathrm{Mu}-$ nicipal de Saúde do Rio de Janeiro e do Hospital Universitário Pedro Ernesto (Universidade do Estado do Rio de Janeiro), os quais facilitaram sobremaneira a obtenção dos dados que originaram este artigo. Agradecimento especial também ao Dr. Alexandre Trajano e à Dra. Mariza Miranda Theme Filha, pela valiosa contribuição ao longo do processo. 


\section{Referências}

1. World Health Organization. World health statistics 2011. Geneva: World Health Organization; 2011. http://www.who.int/whosis/whostat/EN WHS2011_Full.pdf (acessado em 19/Jun/2011).

2. Victora CG, Aquino EM, Leal MC, Monteiro CA, Barros FC, Szwarcwald CL. Maternal and child health in Brazil: progress and challenges. Lancet 2011; 377:1863-76.

3. Ronsmans C, Filippi V. Beyond the numbers: reviewing maternal deaths and complications to make pregnancy safer. Geneva: World Health Organization; 2004

4. Stones W, Lim W, Al-Azzawi F, Kelly M. An investigation of maternal morbidity with identification of life-threatening 'near miss' episodes. Health Trends 1991; 23:13-5.

5. Say L, Pattinson RC, Gulmezoglu AM. WHO systematic review of maternal morbidity and mortality: the prevalence of severe acute maternal morbidity (near miss). Reprod Health 2004; 1:3.

6. Say L, Souza JP, Pattinson RC. Maternal near miss: towards a standard tool for monitoring quality of maternal health care. Best Pract Res Clin Obstet Gynaecol 2009; 23:287-96.

7. Bacci A, Lewis G, Baltag V, Betran AP. The introduction of confidential enquiries into maternal deaths and near-miss case reviews in the WHO European Region. Reprod Health Matters 2007; 15:145-52.

8. Baskett T, O'Connell C. Severe obstetric maternal morbidity: a 15-year population-based study. J Obstet Gynaecol 2005; 25:7-9.

9. Wen SW, Huang L, Liston R, Heaman M, Baskett T, Rusen ID, et al. Severe maternal morbidity in Canada, 1991-2001. CMAJ 2005; 173:759-64.

10. Penney G, Brace V. Near miss audit in obstetrics. Curr Opin Obstet Gynecol 2007; 19:145-50.

11. Souza JP, Cecatti JG, Parpinelli MA, Sousa MH, Serruya SJ. Revisão sistemática sobre morbidade materna near miss. Cad Saúde Pública 2006; 22: 255-64.

12. Mantel GD, Buchmann E, Rees H, Pattinson RC. Severe acute maternal morbidity: a pilot study of a definition for a near-miss. Br J Obstet Gynaecol 1998; 105:985-90.

13. Cantwell R, Clutton-Brock T, Cooper G, Dawson A, Drife J, Garrod D, et al. Saving mothers' lives: reviewing maternal deaths to make motherhood safer: 2006-2008. The Eighth Report of the Confidential Enquiries into Maternal Deaths in the United Kingdom. BJOG 2011; 118 Suppl 1:1-203.

14. The Confidential Enquiry into Maternal and Child Health. Saving mothers' lives: reviewing maternal deaths to make motherhood safer: 2003-2005. The Seventh Report of the Confidential Enquiries into Maternal Deaths in the United Kingdom. London: Confidential Enquiry into Maternal and Child Health; 2007.

15. Sousa MH, Cecatti JG, Hardy EE, Amaral E, Souza JP, Serruya SJ. Sistemas de informação em saúde e monitoramento de morbidade materna grave e mortalidade materna. Rev Bras Saúde Matern Infant 2006;6:161-8.
16. Roberts CL, Cameron CA, Bell JC, Algert CS, Morris JM. Measuring maternal morbidity in routinely collected health data: development and validation of a maternal morbidity outcome indicator. Med Care 2008; 46:786-94

17. Sousa MH, Cecatti JG, Hardy EE, Serruya SJ. Severe maternal morbidity (near miss) as a sentinel event of maternal death. An attempt to use routine data for surveillance. Reprod Health 2008; 5:6.

18. Allen VM, Campbell M, Carson G, Fraser W, Liston RM, Walker M, et al. Maternal mortality and severe maternal morbidity surveillance in Canada. J Obstet Gynaecol Can 2010; 32:1140-6.

19. Bittencourt SA, Camacho LA, Leal MC. O Sistema de Informação Hospitalar e sua aplicação na saúde coletiva. Cad Saúde Pública 2006; 22:19-30.

20. Bittencourt SA, Camacho LA, Leal MC. A qualidade da informação sobre o parto no Sistema de Informações Hospitalares no Município do Rio de Janeiro, Brasil, 1999 a 2001. Cad Saúde Pública 2008; 24:1344-54

21. Veras CMT, Martins MS. A confiabilidade dos dados nos formulários de Autorização de Internação Hospitalar (AIH), Rio de Janeiro, Brasil. Cad Saúde Pública 1994; 10:339-55.

22. Lobato G, Reichenheim ME, Coeli CM. Sistema de informações hospitalares do Sistema Único de Saúde (SIH-SUS): uma avaliação preliminar do seu desempenho no monitoramento da doença hemolítica perinatal Rh(D). Cad Saúde Pública 2008; 24:606-14.

23. World Health Organization. Evaluating the quality of care for severe pregnancy complications: the WHO near-miss approach for maternal health. Geneva: World Health Organization; 2011.

24. Ministério da Saúde. Portaria no 2.816/GM de 29 de maio de 1998. Determina que, no Programa de Digitação de Autorização de Internação Hospitalar - SISAIH01 - seja implantada crítica visando ao pagamento de percentual máximo de cesarianas em relação ao total de partos por hospital. Diário Oficial da União 1998; 2 jun.

25. Ministério da Saúde. Portaria no 94/SAS de 16 de julho de 1998. Determina a AIH sistemática de apresentação e rejeição das AIHs de parto. Diário Oficial da União 1998; 20 jul.

26. Ministério da Saúde. Portaria no 865/GM de 3 de julho de 1999. Redefine os limites de que trata o item 1 da PRT/GM/MS № 2.816, de 29/05/98, publicada no DO 103, de 2/7/98. Diário Oficial da União 1999; 7 jul.

27. Ministério da Saúde. Portaria no 466/GM de 14 de junho de 2000. Estabelecem limites percentuais máximos de cesarianas, em relação ao número total de partos realizados por hospital; considerando a tendência de queda na realização de cesarianas verificada nos últimos 18 (dezoito) meses em todo o país e a necessidade de consolidar o declínio deste procedimento. Diário Oficial da União 2000; 15 jul. 
28. Ministério da Saúde. Portaria no 426/GM de 4 de abril de 2001. Define para o Distrito Federal e os estados que não aderiram ao pacto na forma proposta na Portaria GM/MS № 466, de 14/6/00, os limites totais de cesáreas para o ano de 2001. Diário Oficial da União 2001; 5 abr.

29. Morse ML, Fonseca SC, Gottgtroy CL, Waldmann CS, Gueller E. Severe maternal morbidity and near misses in a regional reference hospital. Rev Bras Epidemiol 2011; 14:310-22.

30. Mathias TA, Soboll ML. Confiabilidade de diagnósticos nos formulários de autorização de internação hospitalar. Rev Saúde Pública 1998; 32:526-32.

31. Ministério da Saúde. Portaria no 579, de 20 de dezembro de 2001. Aprova a tabela de compatibilidade entre o procedimento realizado e o diagnóstico principal informado, de acordo com a CID-10 no processamento das AIHs do Sistema de Informações Hospitalares. Diário Oficial da União 2001; 26 dez.

32. Health Canada. Special report on maternal mortality and severe morbidity in Canada. Enhanced surveillance: the path to prevention. Ottawa: Minister of Public Works and Government Services Canada; 2004.
33. Coeli CM, Blais R, Costa MC, Almeida LM. Probabilistic linkage in household survey on hospital care usage. Rev Saúde Pública 2003; 37:91-9.

34. Camargo Jr. KR, Coeli CM. Reclink: aplicativo para o relacionamento de bases de dados, implementando o método probabilistic record linkage. Cad Saúde Pública 2000; 16:439-47.

35. Silveira DP, Artmann E. Accuracy of probabilistic record linkage applied to health databases: systematic review. Rev Saúde Pública 2009; 43:875-82.

36. Sousa MH, Cecatti JG, Hardy E, Serruya SJ. Relacionamento probabilístico de registros: uma aplicação na área de morbidade materna grave (near miss) e mortalidade materna. Cad Saúde Pública 2008; 24:653-62.

Recebido em 25/Nov/2011

Versão final reapresentada em 12/Fev/2013

Aprovado em 25/Fev/2013 